OPEN ACCESS

Edited by:

Hongcui Cao,

Zhejiang University, China

Reviewed by:

Muhammad IIfan,

Aga Khan University, Pakistan

Jiong Yu,

Zhejiang University, China

*Correspondence:

Carla Maria Irene Quarato carlamariairene.quarato@gmail.com

Specialty section:

This article was submitted to Infectious Diseases - Surveillance,

Prevention and Treatment, a section of the journal

Frontiers in Medicine

Received: 05 August 2021 Accepted: 11 November 2021

Published: 10 December 2021

Citation:

Rea G, Sperandeo M, Lieto R, Bocchino M, Quarato CMI, Feragalli B,

Valente T, Scioscia G, Giuffreda E,

Foschino Barbaro MP and Lacedonia D (2021) Chest Imaging in the Diagnosis and Management of

Pulmonary Tuberculosis: The

Complementary Role of Thoraci

Ultrasound. Front. Med. 8:753821.

doi: 10.3389/fmed.2021.753821

\section{Chest Imaging in the Diagnosis and Management of Pulmonary Tuberculosis: The Complementary Role of Thoraci Ultrasound}

\author{
Gaetano Rea ${ }^{1}$, Marco Sperandeo ${ }^{2}$, Roberta Lieto ${ }^{1}$, Marialuisa Bocchino ${ }^{3}$, \\ Carla Maria Irene Quarato ${ }^{4 *}$, Beatrice Feragalli ${ }^{5}$, Tullio Valente ${ }^{1}$, Giulia Scioscia ${ }^{4}$, \\ Ernesto Giuffreda ${ }^{4}$, Maria Pia Foschino Barbaro ${ }^{4}$ and Donato Lacedonia ${ }^{4}$ \\ ${ }^{1}$ Department of Radiology, Azienda Ospedaliera dei Colli-Cotugno and Monaldi Hospital, Naples, Italy, ${ }^{2}$ Department of \\ Medical Sciences, Unit of Interventional and Diagnostic Ultrasound of Internal Medicine, Istituto di Ricovero e Cura a \\ Carattere Scientifico (IRCCS) Fondazione Casa Sollievo della Sofferenza, San Giovanni Rotondo, Foggia, Italy, ${ }^{3}$ Respiratory \\ Medicine Unit, Department of Clinical Medicine and Surgery, Federico II University, Naples, Italy, ${ }^{4}$ Department of Medical and \\ Surgical Sciences, Institute of Respiratory Diseases, Policlinico Universitario "Riuniti" di Foggia, University of Foggia, Foggia, \\ Italy, ${ }^{5}$ Department of Medical, Oral and Biotechnological Sciences - Radiology Unit "G. D'Annunzio", University of \\ Chieti-Pescara, Chieti, Italy
}

Tuberculosis (TB) is a severe infectious disease that still represents a major cause of mortality and morbidity worldwide. For these reasons, clinicians and radiologists should use all the available diagnostic tools in the assessment of the disease in order to provide precise indications about starting an anti-tubercular treatment and reduce risk of TB transmission and complications especially in developing countries where the disease is still endemic. As TB mycobacteria are mainly transmitted through respiratory droplets, the pulmonary parenchyma is usually the first site of infection. As a result, chest imaging plays a central role in the diagnostic process. Thoracic ultrasound (TUS) is a portable, non-invasive, radiation-free, and cost-contained technology which could be easily available in resource-limited settings. This perspective article focuses on the potential role of TUS in the diagnosis and management of patients with pulmonary TB. Unfortunately, there are still insufficient evidence and too contrasting data to judge TUS as an appropriate diagnostic method for the screening of the disease. Despite this, TUS may have a useful role in identifying pleural and anterior pericardial effusions or in the identification of abscesses of the anterior chest wall and paraspinal collections in low- and middle-income settings. In addition, TUS seems to have a milestone role in guiding minimally invasive interventional procedures, such as placement of chest tubes, drainage of loculated collections, thoracentesis and pericardiocentesis, and percutaneous biopsy of subpleural pulmonary consolidations or pleural plaques.

Keywords: pulmonary tuberculosis, diagnosis and management, chest imaging, chest X-ray (CXR), chest computed tomography (chest CT), thoracic ultrasound (TUS) 


\section{INTRODUCTION}

Tuberculosis (TB) is a severe infective disease that still represents a "world emergency" despite large investments in care over the past three decades. According to the latest report by the World Health Organization (WHO), it is estimated that in 2019, there were 130 new cases of TB per 100,000 population globally. Most of the new cases are found in Asia and Africa (1). Without appropriate treatment, the mortality rate from $\mathrm{TB}$ is high. Moreover, an untreated TB implies, above all, several complications and sequelae that, in the short or long term, are capable of leading to functional and morphological, focal, or diffuse alterations, severely impacting patients' outcome. These include lung post-infectious fibrosis, fibrothorax, bronchiectasis, cavitations, calcific granulomas, dense fibrotic bands, and areas of air-trapping due to post-infectious obstructive bronchiolitis, broncho-pleural fistulas, and lung cancer (scar cancer) (2).

Causative agents of $\mathrm{TB}$ are acid-fast aerobic bacilli belonging to the Mycobacterium Tuberculosis Complex (MTC), mainly including $M$. tuberculosis [and, to a lesser extent, $M$. africanum (3)]. The main gateway of infection in humans is the respiratory tract, by inhalation of aerosolized droplets containing mycobacterium bacilli. These droplets, coming from secretions of the respiratory tract of infected subjects, may remain suspended in the air for a long time, and, given their size, are able to reach small airways and the alveolar compartment (4).

The clinical course and the eventual different manifestations of TB infection mainly depend on immune status of the individual, genetic, and charge of exposure to the microorganism. On average, $5-10 \%$ of infected subjects will develop active TB disease over the course of their lives, usually within the first $2-5$ years after initial infection $(5,6)$. The biggest risk factors for TB reactivation include advanced age, malnutrition, cancer, impaired immunity, AIDS, diabetes, and immunosuppressive treatments, including anti-TNF- $\alpha$ therapies $(7,8)$. A tuberculin skin test (TST) or interferon-gamma release assay (IGRA) can be used for the screening of latent TB. However, these tests can lead to false-negative results, particularly in young children and immune-compromised individuals. "Active" tuberculosis is diagnosed by isolating mycobacterium bacilli from bodily secretions.

Although TB is capable of affecting any organ in the human body, the first site of TB mycobacteria infection is usually the pulmonary parenchyma. As a consequence, chest imaging plays an important first line role for an early diagnosis of the disease. Chest X-ray remains the first instance method for detecting a suspected tuberculosis, although the exam may be normal or show only mild or nonspecific findings in patients with active disease. Chest CT, with or without contrast enhancement, may be helpful for better characterization of radiographic findings by helping to distinguish between previous inactive and active disease or in detecting alterations that cannot be assessed on standard radiograph. "Active" disease is generally characterized by the presence of centrilobular nodules, tree-in-bud pattern, thickwalled cavities, consolidations, miliary nodules, pleural effusions, or necrotic lymphadenopathy (9). "Inactive" tuberculosis is characterized by 6-month stable alterations, including scarring (i.e., peri-bronchial fibrosis, bronchiectasis, and architectural distortion) and nodular opacities (i.e., calcified granulomas and calcified lymph nodes) $(10,11)$. Anyhow, as clinical and radiological signs of TB may mimic those of many other diseases (i.e., lymphoma and other neoplasms or granulomatous diseases and such as sarcoidosis), reaching a specific diagnosis may be challenging (12). Furthermore, in patients with latent TB infection it is not uncommon to detect fibronodular changes especially in the lung apices, making the distinction between active from inactive disease very difficult.

In the last years, thoracic ultrasound (TUS) has gained great interest from both clinicians and radiologists as a useful diagnostic tool for the study of many pleuro-pulmonary conditions (13). Although TUS examination has not yet a standardized role in the diagnosis and management of pulmonary $\mathrm{TB}$ in international guidelines, this imaging technology is advantageous in terms of non-invasiveness and cost-containment, is suitable for a quick real-time evaluation, and is a portable technology readily available for all clinicians in all hospital wards. This makes TUS a potentially useful diagnostic tool for the diagnosis of pulmonary $\mathrm{TB}$, particularly in geographic areas where the access to radiological or laboratory tests is limited. Moreover, TUS has the advantages of not exposing children and pregnant women to ionizing radiation, also representing a diagnostic method that allows considerable improvement in safety for patients who need frequent follow-ups.

The aim of this perspective article is to discuss about the potential role of TUS in the field of tuberculosis diagnosis and management.

\section{IMAGING FINDINGS}

Imaging findings of chest $\mathrm{TB}$ have been classified in lymphadenopathy, parenchymal disease, pleural effusion, pericardial effusion, miliary TB, abscesses of the chest wall, and paraspinal collections. Existing literature and main field of TUS application for each finding have been analyzed and discussed.

\section{LYMPHADENOPATHY}

Lymph node enlargement is seen in up to $96 \%$ of children and $43 \%$ of adults, being typically unilateral and right-sided (14). The most affected lymph node stations are the hilum and right paratracheal region. Although lymphadenopathy is usually associated with other manifestations of $\mathrm{TB}$, it may be the sole radiographic feature. This occurs more common in children $(15,16)$.

Given the advantage of no exposure to ionizing radiation, TUS have been suggested for the assessment of mediastinal lymphadenopathy in children. In 2004, Bosch-Marcet et al. (17) reported success in evaluation of enlarged lymph nodes (with round or oval hypoechoic appearance) in the paratracheal region and aortopulmonary window, because of a good acoustic view. However, ultrasound was not a good technique for assessing hilar involvement, because the hila are surrounded by air in 
the lungs and only large lymph nodes were accessible in the subcarinal region due limitations in the sonographic access (e.g., the esophagus). Despite these limitations, the reported diagnostic performance of TUS in assessing mediastinal lymphoadenopathy was $90.5 \%$. Later, the same group of authors proposed TUS also as an effective non-invasive method for the control and follow-up of mediastinal lymph nodes of children receiving antituberculous chemotherapy (18). More recent studies showed contrasting result, with sensitivity values ranging from 19 to $40 \%(19,20)$. Despite mediastinal lymph nodes enlargement can also be seen in children with other lower respiratory tract infection, the study of Heuvelings et al. (19) highlighted a moderate specificity (71.9\%) for this TUS finding.

If the data relating to the use of TUS are contrasting and do not allow to draw clear conclusions, endobronchial ultrasoundguided transbronchial needle aspiration (EBUS-TBNA) is instead an established minimally invasive method for the assessment of mediastinal/hilar lymphadenopathy in adult with TB. The diagnostic accuracy of the procedure ranges from 68 to $94 \%$ among the various studies (21-27). More limited literature is available on the utility of EBUS-TBNA in children, with a diagnostic accuracy ranging from 36 to $100 \%$ (28-32). Some authors reported that the finding of a heterogeneous echotexture at EBUS or the identification of coagulation necrosis on the granulomatous biopsy could help in the differential diagnosis of tuberculosis compared to other lymph node pathologies, such as sarcoidosis or lymphoma $(33,34)$. The addition of TB cultures and a nucleic acid amplification (NAA) test, such as the Xpert $\mathrm{MTB} / \mathrm{RIF}$ assay, can increase the specificity for the diagnosis of mediastinal tuberculous lymphadenopathy and its drug-resistant form (35).

\section{PARENCHYMAL DISEASE}

In studies examining adult patients with pulmonary $\mathrm{TB}$, the most frequent ultrasound parenchymal findings were subpleural nodules and lung consolidations, while the detection rate of cavitations was low (36-40). A recent systematic review calculated for TUS a sensitivity ranging from 72.5 to $100.0 \%$ in the assessment of subpleural nodules and from 46.7 to $80.4 \%$ in the detection of consolidations. On the other hand, TUS sensitivity for cavitations ranged from 4.0 to $30.0 \%$ (41).

Only the study from Heuvelings et al. (19) provided detailed data on TUS findings in pediatric pulmonary TB. Consolidations were the most common parenchymal alteration in children with pulmonary TB and TUS showed a sensitivity of $45.6 \%$ in their detection.

Lung consolidations are visible on ultrasound only if they are faced to the superficial pleura. In this context, TUS may be used in assessing the response to therapy during the followup of the patients examined, thus configuring a suitable and repeatable alternative to further radiation exposure, that is useful specially in children and pregnant women $(19,42-44)$. However, lung lesions that are not adjacent to the pleura cannot be imaged due to the subtotal reflection that intrapulmonary air determines on the pathway of the ultrasound beam $(45,46)$.
TUS sensitivity for the detection of cavitations appears low in all the studies probably due to the high number of lesions not reaching the pleura. Moreover, the differential diagnosis between the consolidations can be really wide due to the similarities of ultrasound appearance between benign, borderline, or malignant lesions, and TUS specificity in the distinction of consolidation changes (i.e., supervening processes of necrosis, fibrosis, and calcification) is low $(42,45,46)$. According to the study from Montuori et al. (39), in adult with pulmonary TB, the specificity of subpleural nodules, lung consolidations, and cavitations were $66.7,25.3$, and $89.3 \%$, respectively. According to Heuvelings et al. (19) the specificity of lung consolidations in children was 53.3\%.

Bronchogenic spread may be a complication. In such cases, chest $\mathrm{CT}$ allows the identification of multiple micronodules with a centrilobular distribution associated to sharply margined linear branching opacities in the context of the small airways ("treein-bud" sign). The histopathological correlate of centrilobular nodules is the presence of granulomatous inflammation and caseous necrosis within and around terminal and respiratory bronchioles, while peripheral linear branching opacities correspond to tuberculous bronchitis of the small airways (10). These findings are considered indicative of active tuberculosis (47). The "tree-in-bud" pattern usually involves lower lung lobes as centrilobular nodules are peripheral, but spare the subpleural lung (47). Clearly, a diagnosis of small airway disease cannot be carried out with TUS that, in this context, may show only the presence of irregular signs of interface, such as pinching and irregularities of the pleural line and non-specific artifacts including comet tails and ring down (B-lines) (48).

Although common TUS findings of pulmonary TB are not enough specific, this imaging modality can still be helpful in US-guided transthoracic cutting biopsy for histological and microbiological characterization $(45,49,50)$. In the literature, biopsies of TB lesions resulted in moderate to high rates of success, ranging from 77 to $90 \%(36,51)$.

\section{PLEURAL EFFUSION}

Pleural effusion (usually resulting from hypersensitivity to mycobacterium antigens) is typically unilateral $(14,16)$. On Chest $\mathrm{X}$-ray, this finding is usually associated with parenchymal disease. Pleural effusions can remain stable in size for many years, develop septations due to fibrin strands, and result in residual pleural thickening and calcification (14).

Thoracic ultrasound (TUS) is a high effective way in detecting pleural effusion even of modest entity, resulting ultrasound to perform better than chest X-ray. Furthermore, TUS is a fast and useful method not only in differentiating effusion from consolidated lung (whereas both appear "white" on chest radiographs), but also in evaluating the "quality" of liquid alterations. Pleural effusion on ultrasound can appear as anechoic (black), complex non-septated (black with white strands), complex septated (black with white septa), or homogeneously echogenic (white) (52). In general, the presence of homogeneous echogenic effusion suggests corpuscular effusion (i.e., hemorrhage or empyema), whereas 
an anechogenic effusion might be transudative. Since 1989, several studies demonstrated that TUS can be useful in detecting sepimentations, loculations, and thickenings of the pleura in adult patients with pulmonary TB (53-56). However, these studies did not provide data on TUS sensitivity compared to radiographic "gold standard" methods.

According to a recent study from Zhou et al. (57), B-mode ultrasound was less effective than Chest CT in assessing the thickening of the parietal pleura, but there were no substantial differences for pleural effusions and pleural calcifications. The sensitivity values of TUS in detecting pleural effusions, pleural calcifications, and pleural thickenings compared to Chest CT were $77.69,74.01$, and $2.77 \%$. The sensitivity and specificity of the combined three signs in assessing TB pleurisy was 85.51 and $72.34 \%$, respectively. Moreover, although specific data in TB patients are not yet available in the current literature, ultrasound showed a higher sensitivity than chest X-ray and CT scan in detecting fibrin strands and multi-loculation that develop in protein-rich exudative effusions $(58,59)$ (Figures 1A-D).

In addition, TUS is a particularly useful real-time method in guiding thoracentesis needle for risk-free sampling of liquid on which cytologic examination (i.e., lymphocytic effusions are suggestive) and chemical analysis [i.e., determination of pleural fluid adenosine deaminase (ADA) level] can be performed (60, 61) (Figure 1E). Chen et al. (62) found that a complex septate pattern at TUS is a useful predictor of TB in lymphocyterich exudative pleural effusions. A chest tube for evacuative thoracentesis can be safely inserted under TUS guide for the drainage of loculated collections $(45,52)$.

If the results of fluid analysis are not definitive, TUS may be used for transthoracic sample of pleural specimens that can be examined for the histological assessment of granulomas and can be cultured for organisms $(63,64)$. Ultrasound-assisted Abrams' needle biopsy still seems to be the better choice in allowing the diagnosis of pleural tuberculosis compared to ultrasound-guided Tru-Cut needle biopsy (65-67). Sun et al. (68) showed that the Xpert MTB/RIF assay may increase the diagnostic sensitivity for TB in histological pleura samples obtained through contrast enhanced ultrasound (CEUS) guided biopsy. CEUS, on turn, demonstrated to be an efficient, minimally invasive, and safe method for guide biopsy.

\section{PERICARDIAL EFFUSION}

Tuberculosis (TB) is an important cause of pericardial effusions, especially in HIV-infected patients and children. Therefore, an extensive TUS examination, including also a cardiac evaluation, may be an useful cost-effective and radiation-free method to diagnose pericardial effusions in patients with HIV-associated TB and children, especially in geographic areas where TB is endemic (69-73). Ultrasonographically, pericardial effusion appears as an anechoic black stripe around the heart separating the visceral and parietal pericardium. Although Zhou et al. (57) reported that CT examination can more accurately show pericardial effusion in patients with tuberculous pleuritis compared with B-mode ultrasound, pericardial ultrasound examinations showed to be a valuable supplementary investigations in the diagnosis of suspected extrapulmonary or disseminated TB in South Africa (74-76). Ultrasound may also be employed as a safe and costeffective guide for pericardiocentesis in low and middle income settings $(77,78)$. Furthermore, ultrasound follow-up may be very useful for monitoring treatment response in children with extrapulmonary TB (73).

\section{MILIARY TB}

Miliary pulmonary TB is a severe form of disease that occurs in $\sim 1-7 \%$ of TB. It mainly affects the elderly, immunocompromised hosts, and infants. The typical feature of this disease, on both chest X-ray and chest HRCT, is the presence of hundreds of minutes "millet grain" nodules without a preference for anatomical lung location and distributed in both lungs from the apexes to the bases. On High Resolution Chest Tomography (HRCT), many of these nodules are close to fissures and pleural layers with a typical random distribution (14). This pattern is caused by the massive hematogenous dissemination of TB. TUS may highlight slight or marked irregularity of the hyperechoic pleural line that appears as blurred, fragmented, and thickened from the apex to the base in each lung. Moreover, tiny subpleural nodules in miliary TB can determine a non-specific ultrasound pattern characterized by an increased number of discrete B-lines or a blurred or irregular coalescence of multiple artifacts (i.e., Blines, comet tails, A-lines in the same scan image) $(37,38,40,79)$. However, these ultrasound aspects do not allow to differentiate military TB from other HRCT interstitial lung patterns [i.e., Usual Interstitial Pneumonia (UIP), Non-Specific Interstitial Pneumonia (NSIP), Chronic Hypersensitivity Pneumonia (CHP) or others] (80). The marked irregularity of the pleural line, with a thickened, fragmented, and/or blurred appearance, may suggest also an aggressive or advanced stage of fibrotic disease (81). As a result, TUS examination alone may be dangerous and confounding. Only clinical evaluation, laboratory data, and more confidential radiological assessment (Chest X-ray and HRCT) with a correct multidisciplinary approach can allow a correct diagnosis (Figures 2A,B).

\section{TB ABSCESSES}

Due to the systemic nature of tuberculosis, the infection can involve numerous parts of the body, including ribs, sternum, and soft tissue of the chest wall. Typical presentations include abscesses and chronic sinus formation, which are often secondary to lung, pleura, or mediastinal TB. Similarly, iliopsoas abscess is a common complication of Pott's spine (vertebral tuberculosis) in developing countries where the tuberculosis is still an endemic disease (82). TUS, with B-mode examination, may image TB abscesses as hypoechoic areas, with varying degrees of internal heterogeneity (83). Color-Power Doppler well highlights the possible presence of vascular signals (Figures 2C,D). TUS guided percutaneous drainage represents a safe and radiation-free modality of treatment for abscesses of the anterior chest wall 

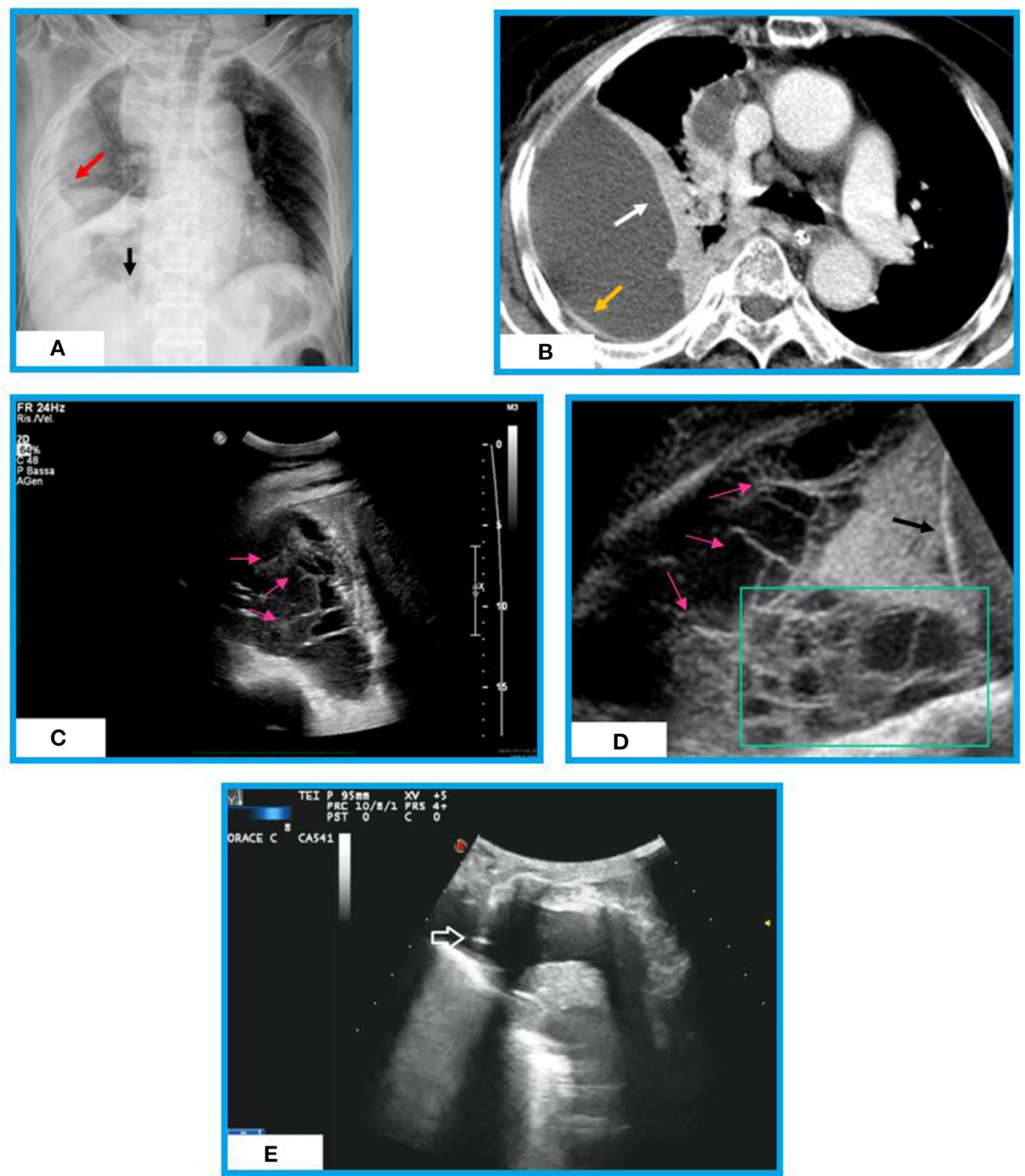

FIGURE 1 | Chest X-ray, chest CT and Ultrasound appearances of an organized effusion in a patient with post-primary TB. (A) Chest X-ray shows a right pulmonary opacity that is not in the gravity dependent location (red arrow). We are able to see the diaphragm medially (black arrow). No septation is seen. (B) Chest CT shows a large loculated right pleural effusion surrounded by a thickened pleural wall (yellow arrow) and lung atelectasis (white arrow). No septation is assessed inside. (C) Thoracic ultrasound (TUS) scan reveals presence of multiple fibrin strands (i.e., thin, mobile, linear hyperechoic structures, pink arrows) forming a septated pleural effusion (complex US aspect). (D) TUS lower thoracic view (diaphragm, black arrow) of the pleural effusion showing multiple septations (pink arrows) and loculations (green box). (E) TUS scan during ultrasound-guided thoracentesis allowing real-time visualization of the needle (white arrow) in an anechoic pleural effusion.

and paraspinal collections, allowing also to clarify their nature $(84,85)$.

\section{DISCUSSION}

For a screening method suitable for pulmonary TB, the WHO recommends a minimum of $90 \%$ sensitivity and $70 \%$ specificity (86). The analysis of the available literature shows that there is still insufficient evidence to judge the diagnostic accuracy of TUS for a screening purpose in TB. The sensitivity of TUS findings between the studies is too varied. This variability may be due to different setting of ultrasound scanners and variable protocols of image acquisition or may imply operator dependence. Furthermore, under optimal conditions, ultrasounds are able to highlight only the $70 \%$ of the pleural surface and everything that closely faces this surface $(45,87)$. Some authors seem to display excessive confidence in the ultrasound examination. As a first step in proper TUS scanning, it is crucial that the operator is accurately trained and has a good understanding of ultrasound physics in order to be able to correctly set-up the ultrasound scanner implementing the diagnostic capabilities of the exam $(45,46)$. 

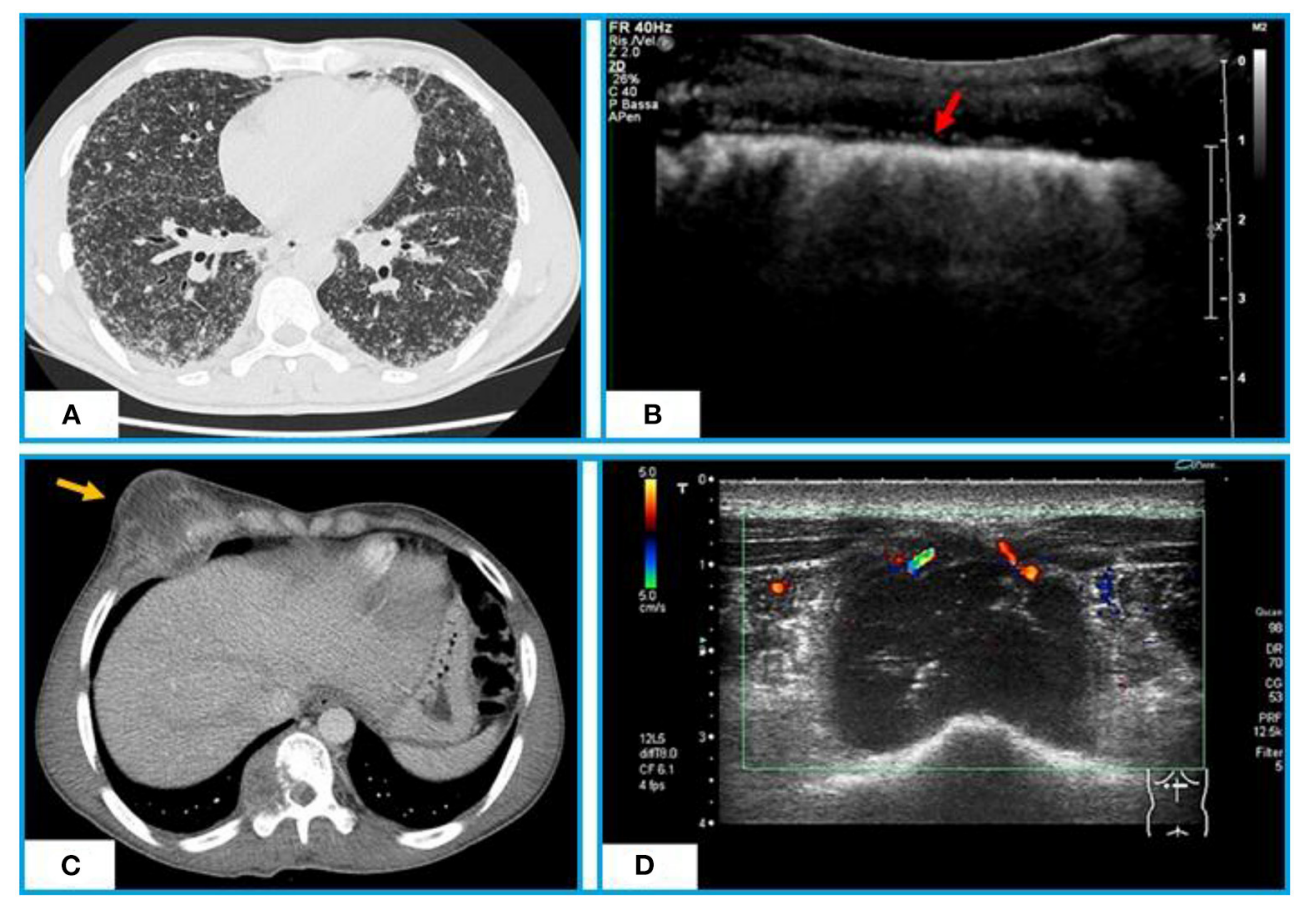

FIGURE 2 | Chest CT and TUS appearance of miliary TB. (A) Axial CT scan shows hundreds of minutes "millet grain" nodules with a typical random distribution. (B) TUS highlights a marked irregularity of the hyperechoic pleural line (red arrow), that appears as blurred and fragmented. Chest CT and TUS appearance of a tuberculous abscess of the anterior chest wall. (C) Axial CT scan with contrast shows a tuberculous abscess with a low-attenuation central necrotic component and capsular ring enhancement extending from the pleura to right chest wall and infiltrating the rib and soft tissues. (D) TUS scan shows a hypoechoic collection in right antero-lateral chest wall with internal echo-debris and no Color-Power Doppler inner signal.

Moreover, the operator should evaluate the patient in terms of physical constitution (i.e., obesity and conformation of the rib cage) and the presence of conditions potentially limiting the examination (i.e., obliged decubitus and presence of dyspnoea) (88). To date, a diagnosis of active TB is accurately highlighted exclusively by clinical context, laboratory and microbiological texts, and standard radiologic exams, including chest X-ray and CT. Despite this, the use of TUS may have a good rationale in particular circumstances that include avoiding exposure to ionizing radiation in children, pregnant women, and patients who need frequent follow-ups and cost-containing in countries with few economic availabilities. Anyhow, the milestone in the employment of TUS in chest TB seems to be represented by its role as a guide in minimally invasive interventional procedures.

Due to the paucity and contradiction of available data, no conclusions can be drawn on diagnostic accuracy of TUS in identifying pathological mediastinal lymph nodes in children with suspected pulmonary TB. In 2017, the group of Pool et al. (89) proposed a protocol for imaging mediastinal lymphadenopathy that was employed only in the study from Heuvelings et al. (19) in children with pulmonary TB. Further studies are needed for validation. Contrary, EBUS-TBNA is a widely accepted technique for sampling mediastinal and hilar lymphadenopathies in adult with TB. More studies on the pediatric population would be desirable in order to confirm the few, but encouraging, currently available data.
Regarding parenchymal ultrasound patterns, subpleural nodules were also described in other acute infective or chronic pulmonary diseases, and the ultrasound pattern does not allow to differentiate the nature of the consolidations. Moreover, ultrasound is clearly unable to discriminate between an active or an inactive TB. In the study from Montuori et al. (39), apical consolidations reached a high specificity (92.2\%) but showed a lower sensitivity (45.1\%). Similarly, cavitations showed good specificity (89.3\%) (39), but sensitivity was very low, ranging from 4.0 to $30.0 \%$ in the various studies (37-40). The explanation for these results relies in the fact that ultrasound is able to detect only evident pathological conditions located in the chest wall or at the pleuro-pulmonary interface when not obscured by bone structures of the chest wall. A composite of several TUS findings may provide acceptable sensitivity and specificity, especially in areas without ready access to standard radiologic exams where TB is endemic $(39,40)$. However, more studies in patients with and without TB are required. Despite these limitations, in confirmed cases of pulmonary TB, TUS may be used in the follow-up of subpleural consolidations to assess response to therapy. In such context, TUS may configure a suitable and repeatable alternative to others imaging methods implying radiation exposure, resulting useful especially in children and pregnant women. In addition, US-guided transthoracic cutting biopsy may represent a safe and minimally invasive technique for histological and microbiological characterization 
of TUS-accessible pulmonary consolidations, allowing to rule out other differential diagnosis.

Thoracic ultrasound (TUS) demonstrated good sensitivity in the detection of pleural effusion. A validated context for TUS employment is the qualitative study of pleural effusion with the detection of septated fluid collection. At the same time, TUS represents a useful real-time and risk-free guide for thoracentesis, drainage of loculated effusions and transthoracic biopsy of pleural plaques. In countries with good economic availability, diagnosis can further avail of TB cultures and a nucleic acid amplification (NAA) test. In areas with high tuberculosis prevalence, the diagnosis of pleural TB may be more simply suggested by the finding of lymphocytosis in a pleural effusion showing a complex septated pattern at TUS.

Given the advantageous cost-containment of this imaging technology, other useful applications of TUS in low- and middleincome settings are the detection of pericardial effusions and pericardiocentesis, which may be especially indicated in patients with HIV-associated TB and children. Last, but not least, TUS may serve as a safe and radiation-free method for guided needle aspiration/drainage of abscesses involving the anterior chest wall and paraspinal collections.

The main limitation of this summary report assessing the role of TUS in pulmonary TB is that, in the absence of controlled studies on large series of TB patients, even TUS usefulness in guiding interventional maneuvers can only be supported by small

\section{REFERENCES}

1. WHO. Global Tuberculosis Report (2020). Available online at: https://www. who.int/publications/i/item/9789240013131 (accessed April 21, 2021).

2. Kim HY, Song K-S, Goo JM, Lee JS, Lee KS, Lim T-H. Thoracic sequelae and complications of tuberculosis. Radiographics. (2001) 21839-58; discussion 859-60. doi: 10.1148/radiographics.21.4.g01j106839

3. de Jong BC, Antonio M, Gagneux S. Mycobacterium africanum-review of an important cause of human tuberculosis in West Africa. PLoS Negl Trop Dis. (2010). 4:e0000744. doi: 10.1371/journal.pntd.0000744

4. Heemskerk D, Caws M, Marais B, Farrar J. "Pathogenesis," In: Heemskerk D, Caws M, Marais B, Farrar J, editors Tuberculosis in Adults and Children, London: Springer (2005) 9-16.

5. Comstock GW, Livesay VT, Woolpert SF. The prognosis of a positive tuberculin reaction in childhood and adolescence. Am J Epidemiol. (1974) 99:131-8. doi: 10.1093/oxfordjournals.aje.a121593

6. Vynnycky E. Lifetime risks, incubation period, and serial interval of tuberculosis. Am J Epidemiol. (2000) 152:247-63. doi: 10.1093/aje/152.3.247

7. CDC. TB, Basic TB Facts: TB Risk Factors. Available online at: https://www. cdc.gov/tb/topic/basics/risk.htm (accessed August 02, 2021).

8. Sanduzzi A, Bocchino M, Atteno M, Costa L, Ponticiello A, Matarese A, et al. Screening and monitoring of latent tubercular infection in patients taking tumor necrosis factor- $\alpha$ blockers for psoriatic arthritis. J Rheumatol Suppl. (2012) 89:82-5. doi: 10.3899/jrheum.120252

9. Skoura E, Zumla A, Bomanji J. Imaging in tuberculosis. Int J Infect Dis. (2015) 32:87-93. doi: 10.1016/j.ijid.2014.12.007

10. Curvo-Semedo L, Teixeira L, Caseiro-Alves F. Tuberculosis of the chest. Eur J Radiol. (2005) 55:158-72. doi: 10.1016/j.ejrad.2005.04.014

11. Diagnostic standards and classification of tuberculosis in adults and children. This official statement of the American Thoracic Society and the Centers for Disease Control and Prevention was adopted by the ATS Board of Directors, July (1999). This statement was endorsed by the Council of the Infectious case series, single case reports and the clinical experience of the authors. Further research is to be encouraged to prove TUS beneficial, especially in countries where TB is endemic.

\section{DATA AVAILABILITY STATEMENT}

The original contributions presented in the study are included in the article/supplementary material, further inquiries can be directed to the corresponding author.

\section{ETHICS STATEMENT}

Due to the descriptive nature of this manuscript, a written informed consent to participate in a clinical study was not required. The explanatory pictures included in this manuscript are from examinations performed as part of our routine medical practice for which patients signed informed consent. Patients provided informed permission for the images publication. The images have been anonymized to protect patients' privacy.

\section{AUTHOR CONTRIBUTIONS}

GR, MS, and DL contributed to the initial conception ad design of the work. All the authors contributed to draft the manuscript or revise it critically for important intellectual content and read and approved the submitted version.
Disease Society of America, September (1999). Am J Respir Crit Care Med. (2000). 161:1376-95. doi: 10.1164/ajrccm.161.4.16141

12. Cardinale L, Nika L, Teti M, Dalpiaz G, Larici AR, Rea G. Pulmonary tuberculosis in diagnostic imaging: the great mime. Recenti Prog Med. (2018) 109:220-5. doi: 10.1701/2896.29193

13. Buda N, Kosiak W, Wełnicki M, Skoczylas A, Olszewski R, Piotrkowski J, et al. Recommendations for lung ultrasound in internal medicine. Diagnostics. (2020) 10:597. doi: 10.3390/diagnostics10080597

14. Burrill J, Williams CJ, Bain G, Conder G, Hine AL, Misra RR. Tuberculosis: a radiologic review. Radiographics. (2007) 27:1255-73. doi: 10.1148/rg.275065176

15. Leung AN, Muller NL, Pineda PR, FitzGerald JM. Primary tuberculosis in childhood: radiographic manifestations. Radiology. (1992) 182:8791. doi: 10.1148/radiology.182.1.1727316

16. Jeong YJ, Lee KS. Pulmonary tuberculosis: up-to-date imaging and management. AJR Am J Roentgenol. (2012) 191:83444. doi: 10.2214/AJR.07.3896

17. Bosch-Marcet J, Serres-Creixams X, Zuasnabar-Cotro A, Codina-Puig $\mathrm{X}$, Català-Puigbó $\mathrm{M}$, Simon-Riazuelo JL. Comparison of ultrasound with plain radiography and CT for the detection of mediastinal lymphadenopathy in children with tuberculosis. Pediatr Radiol. (2004) 34:895-900. doi: 10.1007/s00247-004-1251-3

18. Bosch-Marcet J, Serres-Créixams X, Borrás-Pérez V, Coll-Sibina MT, GuitetJuliá M, Coll-Rosell E. Value of sonography for follow-up of mediastinal lymphadenopathy in children with tuberculosis. J Clin Ultrasound. (2007) 35:118-24. doi: 10.1002/jcu.20304

19. Heuvelings CC, Bélard S, Andronikou S, Jamieson-Luff N, Grobusch MP, Zar HJ. Chest ultrasound findings in children with suspected pulmonary tuberculosis. Pediatr Pulmonol. (2019) 54:463-70. doi: 10.1002/ppul.24230

20. Moseme T, Andronikou S. Through the eye of the suprasternal notch: point-of-care sonography for tuberculous mediastinal lymphadenopathy in children. Pediatr Radiol. (2014) 44:681-4. doi: 10.1007/s00247-014-2890-7 
21. Navani N, Molyneaux PL, Breen RA, Connell DW, Jepson A, Nankivell $\mathrm{M}$, et al. Utility of endobronchial ultrasound-guided transbronchial needle aspiration in patients with tuberculous intrathoracic lymphadenopathy: a multicentre study. Thorax. (2011) 66:889. doi: 10.1136/thoraxjnl-2011200063

22. Madan K, Mohan A, Ayub II, Jain D, Hadda V, Khilnani GC, et al. Initial experience with endobronchial ultrasound-guided transbronchial needle aspiration (EBUS-TBNA) from a tuberculosis endemic population. J Bronchology Interv Pulmonol. (2014) 21:208-14. doi: 10.1097/LBR.0000000000000080

23. Hassan T, McLaughlin AM, O’Connell F, Gibbons N, Nicholson S, Keane J, et al. Performs well in the diagnosis of isolated thoracic tuberculous lymphadenopathy. Am J Respir Crit Care Med. (2012) 183:1367. doi: 10.1164/ajrccm.183.1.136

24. Sun J, Teng J, Yang H, Li Z, Zhang J, Zhao H, et al. Endobronchial ultrasound-guided transbronchial needle aspiration in diagnosing intrathoracic tuberculosis. Ann Thorac Surg. (2013) 96:20217. doi: 10.1016/j.athoracsur.2013.07.005

25. Gupta N, Muthu V, Agarwal R, Dhooria S. Role of EBUS-TBNA in the diagnosis of tuberculosis and sarcoidosis. J Cytol. (2019) 36:128. doi: 10.4103/JOC.JOC_150_18

26. Wang Y, Zhu H, Yang S, Wang K, Tian PW, Shi JY, et al. The diagnostic value of endobronchial ultrasound-guided needle aspiration biopsy for lung or mediastinal lymph node cancer and tuberculosis. Sichuan Da Xue Xue Bao Yi Xue Ban. (2017). 48:347-51.

27. Erer OF, Erol S, Anar C, Biçmen C, Aydogdu Z, Aktogu S. The diagnostic accuracy of endobronchial ultrasound-guided transbronchial needle aspiration (EBUS-TBNA) in mediastinal tuberculous lymphadenitis. Turk J Med Sci. (2017) 47:1874-9. doi: 10.3906/sag-1606-110

28. Gilbert CR, Chen A, Akulian JA, Lee HJ, Wahidi M, Argento AC, et al. The use of convex probe endobronchial ultrasound-guided transbronchial needle aspiration in a pediatric population: a multicenter study. Pediatr Pulmonol. (2014) 49:807-15. doi: 10.1002/ppul.22887

29. Dhooria S, Madan K, Pattabhiraman V, Sehgal IS, Mehta R, Vishwanath $\mathrm{G}$, et al. A multicenter study on the utility and safety of EBUSTBNA and EUS-B-FNA in children. Pediatr Pulmonol. (2016) 51:10319. doi: 10.1002/ppul.23415

30. Gulla KM, Gunathilaka G, Jat KR, Sankar J, Karan M, Lodha R, et al. Utility and safety of endobronchial ultrasound-guided transbronchial needle aspiration and endoscopic ultrasound with an echobronchoscope-guided fine needle aspiration in children with mediastinal pathology. Pediatr Pulmonol. (2019) 54:881-5. doi: 10.1002/ppul.24313

31. Shanthikumar S, Massie J, Ranganathan S, Schuhmann M, Eberhardt R, Irving LB, et al. Utility of endobronchial ultrasound in assessment of intrathoracic lesions in paediatric patients. Respiration. (2019) 98:3406. doi: 10.1159/000501466

32. Al-Najjar H, Breen R, Santis G, Narayan O. The utility and safety of linear endobronchial ultrasound-guided transbronchial needle aspiration (EBUS-TBNA) in the paediatric population. Eur Respir J. (2020) 55:1902277. doi: 10.1183/13993003.02277-2019

33. Dhooria S, Agarwal R, Aggarwal AN, Bal A, Gupta N, Gupta D. Differentiating tuberculosis from sarcoidosis by sonographic characteristics of lymph nodes on endobronchial ultrasonography: a study of 165 patients. J Thorac Cardiovasc Surg. (2014) 148:662-7. doi: 10.1016/j.jtcvs.2014.01.028

34. Cheng W-C, Chen C-Y, Chen W-C, Liao W-C, Chen C-H, Tu CY. Identification of specific EBUS-TBNA sonographic characteristics for differentiating sarcoidosis from tuberculosis and lymphoma. Eur Respir J. (2019) 54:PA4836. doi: 10.1183/13993003.congress-2019.PA4836

35. Chhajed PN, Vaidya PJ, Mandovra NP, Chavhan VB, Lele TT, Nair R, et al. EBUS-TBNA in the rapid microbiological diagnosis of drug-resistant mediastinal tuberculous lymphadenopathy. ERJ Open Res. (2019) 5:000082019. doi: 10.1183/23120541.00008-2019

36. Yuan A, Yang PC, Chang DB, Yu CJ, Lee LN, Wu HD, et al. Ultrasound guided aspiration biopsy for pulmonary tuberculosis with unusual radiographic appearances. Thorax. (1993) 48:167-70. doi: 10.1136/thx.48.2.167

37. Agostinis P, Copetti R, Lapini L, Monteiro GB, N’Deque A, Baritussio A. Chest ultrasound findings in pulmonary tuberculosis. Trop Doct. (2017) 47:320-8. doi: 10.1177/0049475517709633
38. Wagih KM, Abdel Halim HA, El Sayed El Basuni YM. Chest ultrasound patterns in patients with pulmonary tuberculosis with and without HIV. Egypt J Chest Dis Tuberc. (2020) 69:421. doi: 10.4103/ejcdt_107_19

39. Montuori M, Casella F, Casazza G, Franzetti F, Pini P, Invernizzi C, et al. Lung ultrasonography in pulmonary tuberculosis: a pilot study on diagnostic accuracy in a high-risk population. Eur J Intern Med. (2019) 66:29-34. doi: 10.1016/j.ejim.2019.06.002

40. Fentress M, Ugarte-Gil C, Cervantes M, Rivas D, Moore D, Caliguiri P, et al. Lung ultrasound findings compared with chest X-ray findings in known pulmonary tuberculosis patients: a cross-sectional study in Lima, Peru. Am J Trop Med Hyg. (2020) 103:1827-33. doi: 10.4269/ajtmh.20-0542

41. Bigio J, Kohli M, Klinton JS, MacLean E, Gore G, Small PM et al. Diagnostic accuracy of point-of-care ultrasound for pulmonary tuberculosis: a systematic review. PLoS ONE. (2021) 16:e0251236. doi: 10.1371/journal.pone.0251236

42. Rea G, Sperandeo M, Di Serafino M, Vallone G, Tomà P. Neonatal and pediatric thoracic ultrasonography. J Ultrasound. (2019) 22:12130. doi: 10.1007/s40477-019-00357-6

43. D'Amato M, Rea G, Carnevale V, Grimaldi MA, Saponara AR, Rosenthal E, et al. Assessment of thoracic ultrasound in complementary diagnosis and in follow up of community-acquired pneumonia (CAP). BMC Med Imaging. (2017) 17:52. doi: 10.1186/s12880-017-0225-5

44. Sperandeo M, Tinti MG, Rea G. Chest ultrasound versus chest X-rays for detecting pneumonia in children: why compare them each other if together can improve the diagnosis? Eur J Radiol. (2017) 93:2912. doi: 10.1016/j.ejrad.2017.05.038

45. Sperandeo M, Rotondo A, Guglielmi G, Catalano D, Feragalli B, Trovato GM. Transthoracic ultrasound in the assessment of pleural and pulmonary diseases: use and limitations. Radiol Med. (2014) 119:729-40. doi: 10.1007/s11547-014-0385-0

46. Sperandeo M, Filabozzi P, Varriale A, Carnevale V, Piattelli ML, Sperandeo G et al. Role of thoracic ultrasound in the assessment of pleural and pulmonary diseases. J Ultrasound. (2008) 11:39-46. doi: 10.1016/j.jus.2008.02.001

47. Im JG, Itoh H, Shim YS, Lee JH, Ahn J, Han MC, et al. Pulmonary tuberculosis: CT findings-early active disease and sequential change with antituberculous therapy. Radiology. (1993) 186:653-60. doi: 10.1148/radiology.186.3.8430169

48. Tinti MG, Rea G, Frongillo E, Saponara A, Sperandeo M. The pathologic patterns detectable by transthoracic ultrasonography are only the pleural and subpleural ones and are not specific: why compare them with highresolution computed tomography? J Ultrasound Med. (2018) 37:18478. doi: 10.1002/jum.14510

49. Sperandeo M, Del Colle A, Frongillo E, Rea G, Dimitri L Cipriani C, Lacedonia D. Safety maximization of percutaneous transthoracic needle biopsy with ultrasound guide in subpleural lesions in the evaluation of pulmonary consolidation. Respir Res. (2019) 20:68. doi: 10.1186/s12931-019-1031-0

50. Zhao Z-L, Peng L-L, Wei Y, Li Y, Wang G-M, Yu M-A. The accuracy of ultrasound-guided lung biopsy pathology and microbial cultures for peripheral lung lesions. J Thorac Dis. (2020) 12:858. doi: $10.21037 /$ jtd.2019.12.92

51. Gulati M, Venkataramu NK, Gupta S, Sood BP, Sheena DM, Gupta SK, Suri S. Ultrasound guided fine needle aspiration biopsy in mediastinal tuberculosis. Int J Tuberc Lung Dis. (2000) 4:1164-8.

52. Yang PC, Luh KT, Chang DB, Wu HD, Yu CJ, Kuo SH. Value of sonography in determining the nature of pleural effusion: analysis of 320 cases. Am J Roentgenol. (1992) 159:29-33. doi: 10.2214/ajr.159.1.1609716

53. Martínez OC, Serrano BV, Rodr Romero RÍ. Real-time ultrasound evaluation of tuberculous pleural effusions. J Clin Ultrasound. (1989) 17:40710. doi: 10.1002/jcu.1870170605

54. Akhan O, Demirkazik FB, Ozmen MN, Balkanci F, Ozkara S, Cöplü L, et al. Tuberculous pleural effusions: ultrasonic diagnosis. J Clin Ultrasound. (1992) 20:461-5. doi: 10.1002/jcu.1870200708

55. Ahuja V, Gombar S, Kumar N, Goyal N, Gupta K. Pregnancy with bilateral tubercular pleural effusion: challenges. Trop Doct. (2014) 44:1168. doi: 10.1177/0049475513517117

56. Lai Y-F, Su M-C, Weng H-H, Wu J-T, Chiu C-T. Sonographic septation: a predictor of sequelae of tuberculous pleurisy after treatment. Thorax. (2009) 64:806-9. doi: 10.1136/thx.2008.110197

57. Zhou S, Zhao J, Song X, Zheng $M$, Li H, Pan Y. Imaging manifestations of B-mode ultrasound combined with $\mathrm{CT}$ in tuberculous 
pleuritis patients and the diagnostic value. Exp Ther Med. (2018) 16:2343-8. doi: 10.3892/etm.2018.6471

58. Kearney SE, Davies CWH, Davies RJO, Gleeson FV. Computed tomography and ultrasound in parapneumonic effusions and empyema. Clin Radiol. (2000) 55:542-7. doi: 10.1053/crad.1999.0480

59. Khalil M, Diab HS, Hosny H, Edward E, Thabet E, Emara W, et al. Chest ultrasound versus chest computed tomography for imaging assessment before medical thoracoscopy. Egypt J Bronchol. (2014) 8:14952. doi: 10.4103/1687-8426.145714

60. Ahmed WAW, Rahim MJC, Mohammad N, Fauzi MH, Wahab SFA. Hiding in plain sight: DIAGNOSING pleural tuberculosis using lung ultrasound. Ultrasound. 29:123-7. doi: 10.1177/1742271X20959761

61. Zhai K, Lu Y, Shi H-Z. Tuberculous pleural effusion. J Thorac Dis. (2016) 8:E486. doi: 10.21037/jtd.2016.05.87

62. Chen HJ, Hsu WH, Tu CY, Yu YH, Chiu KL, Hang LW, et al. Sonographic septation in lymphocyte-rich exudative pleural effusions: a useful diagnostic predictor for tuberculosis. J Ultrasound Med. (2006) 25:85763. doi: 10.7863/jum.2006.25.7.857

63. Bahr HM, El-Shafey MH, Hantera MS, Abo-El magd GH, ElBatsh AH. Ultrasound guided needle pleural biopsy in patients with undiagnosed pleural effusion. Egypt J Chest Dis Tuberc. (2014) 63:113-8. doi: 10.1016/j.ejcdt.2013.11.019

64. Zhou X, Jiang P, Huan X, Li W, Chen Y, Gao H, et al. Ultrasound-guided versus thoracoscopic pleural biopsy for diagnosing tuberculous pleurisy following inconclusive thoracentesis: a randomized, controlled trial. Med Sci Monit. (2018) 24:7238-48. doi: 10.12659/MSM.912506

65. Koegelenberg CF, Bolliger CT, Theron J, Walzl G, Wright CA, Louw M, et al. Direct comparison of the diagnostic yield of ultrasound-assisted Abrams and Tru-Cut needle biopsies for pleural tuberculosis. Thorax. (2010) 65:85762. doi: 10.1136/thx.2009.125146

66. Chang DB, Yang PC, Luh KT, Kuo SH, Yu CJ. Ultrasoundguided pleural biopsy with Tru-Cut needle. Chest. (1991) 100:1328-33. doi: 10.1378/chest.100.5.1328

67. Botana-Rial M, Leiro-Fernández V, Represas-Represas C, González-Piñeiro A, Tilve-Gómez A, Fernández-Villar A. Thoracic ultrasound-assisted selection for pleural biopsy with abrams needle. Respir Care. (2013) 58:194954. doi: $10.4187 /$ respcare. 02378

68. Sun W, Zhou Y, Li W, Wang Y, Xiong K, Zhang Z, et al. Diagnostic yield of Xpert MTB/RIF on contrast-enhanced ultrasound-guided pleural biopsy specimens for pleural tuberculosis. Int J Infect Dis. (2021) 108:8995. doi: 10.1016/j.ijid.2021.05.023

69. Weber SF, Saravu K, Heller T, Kadavigere R, Vishwanath S, Gehring $\mathrm{S}$, et al. Point-of-care ultrasound for extrapulmonary tuberculosis in india: a prospective cohort study in HIV-positive and HIV-negative presumptive tuberculosis patients. Am J Trop Med Hyg. (2018) 98:266. doi: 10.4269/ajtmh.17-0486

70. Heller T, Mtemang'ombe EA, Huson MA, Heuvelings CC, Bélard S, Janssen S, et al. Ultrasound for patients in a high HIV/tuberculosis prevalence setting: a needs assessment and review of focused applications for Sub-Saharan Africa. Int J Infect Dis. (2017) 56:229-36. doi: 10.1016/j.ijid.2016.11.001

71. Bobbio F, Di Gennaro F, Marotta C, Kok J, Akec G, Norbis L, et al. Focused ultrasound to diagnose HIV-associated tuberculosis (FASH) in the extremely resource-limited setting of South Sudan: a cross-sectional study. BMJ Open. (2019) 9:e027179. doi: 10.1136/bmjopen-2018-027179

72. Weber S. Tuberculosis and pericarditis in children. Trop Doct. (1999) 29:1358. doi: 10.1177/004947559902900304

73. Bélard S, Heuvelings CC, Banderker E, Bateman L, Heller T, Andronikou S, et al. Utility of point-of-care ultrasound in children with pulmonary tuberculosis. Pediatr Infect Dis J. (2018) 37:637-42. doi: 10.1097/INF.0000000000001872

74. Patel MN, Beningfield S, Burch V. Abdominal and pericardial ultrasound in suspected extrapulmonary or disseminated tuberculosis. South African Med J. (2011) 101:39-42. doi: 10.7196/SAMJ.4201

75. Van Hoving DJ, Kenge AP, Maartens G, Meintjes G. Point-of-care ultrasound predictors for the diagnosis of tuberculosis in HIV-positive patients presenting to an emergency center. J Acquir Immune Defic Syndr. (2020) 83:41523. doi: 10.1097/QAI.0000000000002279
76. Ndege R, Weisser M, Elzi L, Diggelmann F, Bani F, Gingo W, et al. Sonography to rule out tuberculosis in sub-saharan africa: a prospective observational study. Open Forum Infect Dis. (2019) 6:ofz154. doi: 10.1093/ofid/ofz154

77. Heller T, Lessells RJ, Wallrauch C, Brunetti E. Case report: tuberculosis pericarditis with cardiac tamponade: management in the resource-limited setting. Am J Trop Med Hyg. (2010) 83:1311. doi: 10.4269/ajtmh.2010.10-0271

78. Heller T, Wallrauch C, Goblirsch S, Brunetti E. Focused assessment with sonography for HIV-associated tuberculosis (FASH): a short protocol and a pictorial review. Crit Ultrasound J. (2012) 41:1-9. doi: 10.1186/2036-7902-4-21

79. Hunter L, Bélard S, Janssen S, van Hoving DJ, Heller T. Miliary tuberculosis: sonographic pattern in chest ultrasound. Infection. (2016) 44:243-6. doi: 10.1007/s15010-015-0865-8

80. Sperandeo M, Rea G. "Interstitial lung diseases," In: Feletti F, Malta B, Aliverti $\mathrm{B}$, editors. Thoracic Ultrasound and Integrated Imaging, Springer Nature Switzerland AG, 61-82.

81. Lacedonia D, Scioscia G, Giardinelli A, Quarato CMI, Sassani EV, Foschino Barbaro MP, et al. The role of transthoracic ultrasound in the study of interstitial lung diseases: high-resolution computed tomography versus ultrasound patterns: our preliminary experience. Diagnostics. (2021) 11:439. doi: 10.3390/diagnostics11030439

82. Vijayvergia P, Vijayan N, Midha NK, Kumar D, Gopalakrishnan M, Tiwari S, et al. Methicillin-sensitive staphylococcus aureus pyogenic iliopsoas abscesses: a case series from Jodhpur, India. Infect Disord Drug Targets. (2020) 21:e270421187570. doi: 10.2174/1871526520999201103192948

83. Zhang Y, Xu J, He N, Zhang L, Yu X, Yang G. Diagnostic value of highfrequency ultrasonography in chest wall tuberculosis abscess. Chinese J Med Ultrasound (Electronic Ed). (2018) 15:209.

84. Sonhaye L, Amadou A, Gnandi-Piou F, Assih K, Tchaou M, Kolou B, et al. Tuberculous abscess of the chest wall simulate pyogenic abscess. Case Rep Radiol. (2015) 2015:195412. doi: 10.1155/2015/195412

85. Iloanusi NI, Unigwe US, Uche EO, Iroezindu MO, Okafor OC. Pott's disease with extensive bilateral psoas abscesses in a Nigerian woman: an unusual case. Malawi Med J. (2020) 32:168. doi: 10.4314/mmj.v32 i3.10

86. WHO. High-Priority Target Product Profiles for New Tuberculosis Diagnostics. Available online at: https://www.who.int/publications/i/item/WHO-HTMTB-2014.18 (accessed August 02, 2021).

87. Quarato CMI, Venuti M, Sperandeo M. The artificial count of artifacts for thoracic ultrasound: what is the clinical usefulness? J Clin Monit Comput. (2020) 34:1379-81. doi: 10.1007/s10877-020-00484-0

88. Reißig A, Görg C, Mathis G. Transthorakale Sonografie bei der Diagnostik pulmonaler Erkrankungen: ein systematischer Zugang. Ultraschall der Medizin - Eur J Ultrasound. (2009) 30:438-58. doi: 10.1055/s-0028-1109703

89. Pool KL, Heuvelings CC, Bélard S, Grobusch MP, Zar HJ, Bulas D, et al. Technical aspects of mediastinal ultrasound for pediatric pulmonary tuberculosis. Pediatr Radiol. (2017) 47:1839-48. doi: 10.1007/s00247-017-3954-2

Conflict of Interest: The authors declare that the research was conducted in the absence of any commercial or financial relationships that could be construed as a potential conflict of interest.

Publisher's Note: All claims expressed in this article are solely those of the authors and do not necessarily represent those of their affiliated organizations, or those of the publisher, the editors and the reviewers. Any product that may be evaluated in this article, or claim that may be made by its manufacturer, is not guaranteed or endorsed by the publisher.

Copyright (c) 2021 Rea, Sperandeo, Lieto, Bocchino, Quarato, Feragalli, Valente, Scioscia, Giuffreda, Foschino Barbaro and Lacedonia. This is an open-access article distributed under the terms of the Creative Commons Attribution License (CC BY). The use, distribution or reproduction in other forums is permitted, provided the original author(s) and the copyright owner(s) are credited and that the original publication in this journal is cited, in accordance with accepted academic practice. No use, distribution or reproduction is permitted which does not comply with these terms. 